\title{
Application of Newton/GMRES Method to Nonlinear Model Predictive Control of Functional Electrical Stimulation
}

\author{
Naser Mehrabi, Sadegh Tajeddin, Nasser L. Azad, John McPhee \\ University of Waterloo \\ 200 University Ave W, Waterloo, Ontario \\ [nmehrabi, stajeddin, nlashgar, mcphee]@uwaterloo.ca
}

\begin{abstract}
Recent studies have shown that functional electrical stimulation (FES) therapy can improve the motor recovery and range of the motion of stroke patients. The state-of-the-art functional electrical stimulators are open-loop control systems, i.e., the controller is unaware of the patient's posture and progress during the therapy. In this research, we have developed a closed-loop Newton/GMRES nonlinear model predictive controller that measures the patient posture and controls the muscle stimulation to reach a desired posture.
\end{abstract}

Keywords: Functional Electrical Stimulation (FES), Nonlinear Model Predictive Control (NMPC), Newton/GMRES

\section{Introduction}

Disabilities resulting from stroke and spinal cord injuries can greatly affect the patient's ambulation and quality of life. Functional electrical stimulation (FES) is a method used by therapists to restore the natural motion of the affected limbs [1]. FES can activate the nerves innervating a skeletal muscle by injecting an electrical current into the muscles. Recent research studies have shown that by a well-timed sequential stimulation of skeletal muscles, complex actions such as gait [2] can be achieved. Optimal open-loop controllers have commonly been used to find these sequences. However, with no feedback information, these controllers are not robust to disturbances or other uncertainties, such as muscle fatigue.

Recently proposed FES feedback controllers incorporate real-time measurements of the limb orientation and position to overcome the robustness and stability issues. As an example, the sliding mode controller proposed by Lynch et al. [3] provides a guaranteed stability; however, no muscle fatigue criterion has been included in the controller logic. Mohammed et al. [4] developed a linear model predictive control (MPC) applied to an input-output feedback linearized model of a human knee to minimize the trajectory tracking error. The MPC uses quadratic programing to find the optimal dynamics of a linear system for a finite prediction horizon. In contrast to the linear MPC, the nonlinear MPC (NMPC) uses a nonlinear model to provide more accurate prediction of the system behaviour. Kirsch et al. [5] have recently developed a nonlinear model predictive FES controller that adopted the Pontryagin's maximum principle to find the optimal dynamics at each horizon. However, this implementation of NMPC is computationally expensive, and may not be suitable for large systems with many inputs and states; it cannot be used for real-time FES control. Therefore, in this present paper, we have proposed a real-time Newton/Generalized minimal residual (GMRES) nonlinear model predictive controller for FES. To study the capability of this controller, a nonlinear model predictive FES controller is designed and applied to a high-fidelity human knee model to control the knee extension while minimizing the muscular activation.

\section{Dynamical Model}

We have developed two models of the human knee: a high-fidelity model and a simple yet efficient control-oriented model. The high-fidelity model is a detailed model of knee that includes wrapping geometries defining the muscle paths. The control-oriented model is a simplified model to be used at the heart of the FES controller. This model reduces the computational burden to support the real-time iterative calculations within the NMPC.

\subsection{High-Fidelity Model}

A detailed model of lower-extremity including thigh, shank and foot as shown in Figure 1 has been developed in the MapleSim software. This model is in a seated position, where the thigh is assumed to be fixed and connected to the shank via a revolute joint. An electrical stimulator has been attached to the Rectus Femoris (Rfem), which is a major knee extensor. 
In this research, a cylindrical wrapping geometry, as defined in the obstacle-set method, is used to realistically simulate the Rfem wrapping around the knee. This method provides an accurate estimation of the muscle length and moment arm in comparison to other commonly used methods. A modified Hill-type muscle model has been used to simulate the contraction dynamics of Rfem stimulated by low intensity electrical current. This muscle model consists of a contractile element and a parallel-elastic element and assumes an inextensible tendon as the series-elastic element. The knee model parameters are based on the Ferrarian and Pedotti's knee model [2] in which the parameters were identified from several subjects with complete spinal cord injury.

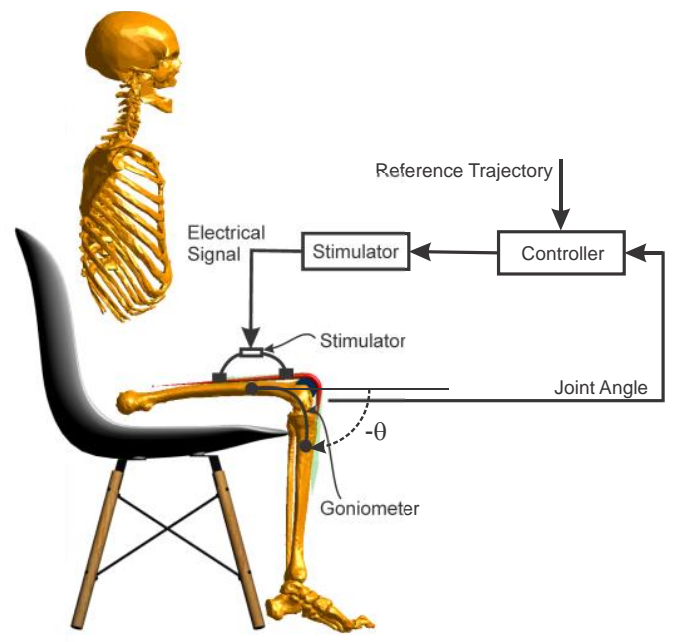

Fig. 1. Schematic views of the high-fidelity model of knee and the FES controller block diagram.

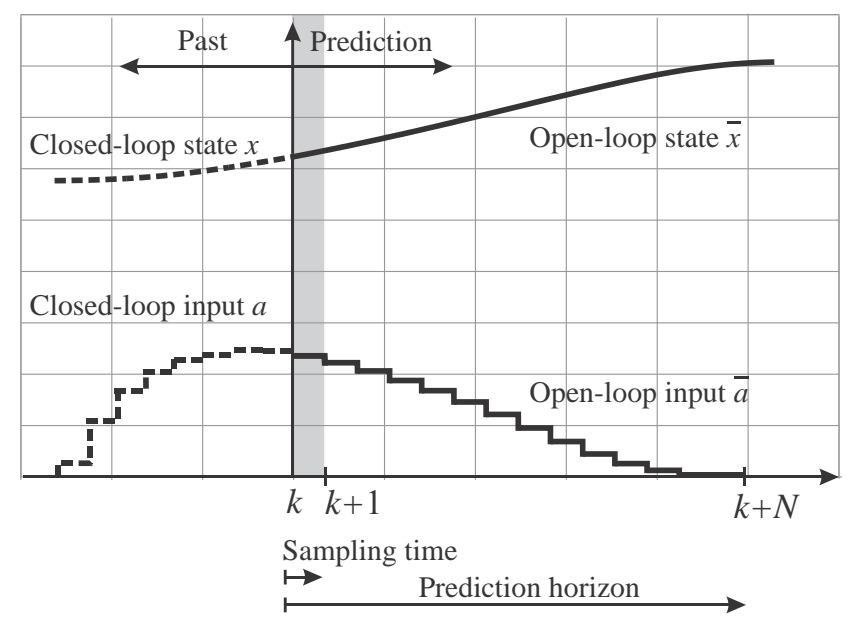

Fig. 2. Schematic view of the nonlinear model predictive control.

The solid lines show the optimal states and control inputs prediction in a given horizon and dashed lines are the past inputs and state trajectories.

\subsection{Control-Oriented Model}

In the control-oriented model, similar to the high-fidelity model, the thigh is resting on the seat and only the shank is rotating about the knee. In this model, the dynamic equation of motion of the shank and foot is described by:

$$
I \ddot{\theta}=T_{\text {Passive }}(\theta, \dot{\theta})+T_{g}+T_{\text {active }}(\theta, \dot{\theta})
$$

where $\theta, \dot{\theta}$ and $\ddot{\theta}$ are respectively the knee angle, angular speed and acceleration, and $I$ is the shank and foot combined moment of inertia. $T_{\text {passive }}, T_{g}$ and $T_{\text {active }}$ are the torques due to passive musculotendon dynamics, gravity and muscle force at the knee, respectively. The passive musculoskeletal torque consists of the stiffness and damping torques [6]:

$$
T_{\text {Passive }}=-\lambda e^{-E \theta}(\theta-\omega)-C \dot{\theta}
$$

where the first term is the stiffness torque in which $\lambda$ and $E$ are the exponential coefficients of the stiffness torque and $\omega$ is the unstretched elastic angle, and the second term is the damping torque where $C$ is the viscous damping coefficient of the knee. The gravitational torque $\left(T_{g}\right)$ about the knee is:

$$
T_{g}=-m g l \cos (\theta)
$$

where $m$ is the total mass of the shank and foot and $l$ is the centre of mass distance from the knee. The active torque is the torque produced by Rectus Femoris about the knee:

$$
T_{\text {active }}=r^{M}(\theta) F^{M}(\theta, \dot{\theta})
$$


where $F^{M}$ is the muscle force calculated from the muscle model and $r^{M}$ is the moment arm of Rfem. The Rfem moment arm is approximated with a $4^{\text {th }}$-order polynomial fitted to the simulation results from the high-fidelity knee model. To simplify the muscle dynamics, the force-velocity dependence of the contractile element is ignored, which is not unreasonable since the desired motion of the leg is relatively slow. Finally, the muscle force can be simplified to:

$$
F^{M}=a(\mathrm{t}) F_{0}^{\max } e^{\left(\frac{l(\theta)-l_{s}}{L_{M}^{o p t} \cos \left(\alpha_{p}\right)}-1\right)^{2} / \gamma} \cos \left(\alpha_{p}\right)
$$

where $l, l_{s}$ and $L_{M}^{o p t}$ are the muscle length, muscle optimal length, and tendon slack length, and $\gamma$ and $\alpha_{p}$ are the shaping factor and muscle pennation angle, respectively. Similar to the moment arm, the muscle length is also approximated with a $4^{\text {th }}$-order polynomial derived from the high-fidelity knee model. Knowing the desired muscle activation $a(\mathrm{t})$, the required electrical stimulation can be easily calculated with a linear mapping from muscle activation to electrical current [4].

\section{GMRES Nonlinear Model Predictive Control}

A nonlinear model predictive controller has been used to control the FES system. NMPC is an advanced control technique that employs a nonlinear control-oriented model to predict the behaviour of the system over a receding horizon, and generates an optimal control sequence. Fast optimization methods such as Newton/GMRES and Continuation/GMRES can significantly reduce the computational time, allowing the real-time implementation of such controllers [7]. From a computational perspective, GMRES-based optimization methods are claimed to be fast as they solve the differential equation once at each time step. In this study, a Newton/GMRES method is implemented, which utilizes a forward difference GMRES algorithm [8] to find Newton's step for solving the Pontryagin's maximum principle problem. The NMPC predicts the optimal dynamics of the system $(\bar{x}, \bar{a})$ over the given prediction horizon (Figure 2 ) by minimizing the following cost function:

$$
\begin{gathered}
J=\sum_{i=1}^{N} w_{1}\left(\theta(i)-\theta_{\text {tgt }}\right)^{2}+w_{2} a^{2}(i) \\
\text { subject to: } 0 \leq a(i) \leq 1
\end{gathered}
$$

where $N$ is the prediction horizon length, and $w_{1}$ and $w_{2}$ are the tracking and effort cost function weights, respectively, $\theta_{t g t}$ is the target knee angle, and $a^{2}$ is the required muscular effort to extend the knee.

\section{Simulation Results}

It is assumed that the knee is initially at rest as shown in Fig.1, where the knee angle is $-80^{\circ}$. A smooth step-like trajectory as shown in Fig 3 has been used to evaluate the performance of the proposed FES controller. First, the performance of the NMPC controller has been investigated and compared to a manually tuned PID controller. Then, the effect of prediction horizon length on the control performance has been studied.

In the first scenario, the tracking to effort ratio $\left(\alpha=\frac{w_{1}}{w_{2}}\right)$ has been varied from 1 to 6 , where $\alpha=1$ means equal weights on the tracking and effort. In these simulations, the NMPC time step has been set to $50 \mathrm{~ms}$ and the prediction horizon is assumed to be 10 steps. As shown in Fig. 3, increasing $\alpha$ improves the tracking performance and simultaneously increases the required muscle activation (see Fig 4). As shown in Fig. 5, the total amount of current delivered through FES electrodes using the NMPC is less than the PID controller. This reduction is due to the flexibility of NMPC in tracking the desired trajectory and the ability to predict the knee dynamics. 


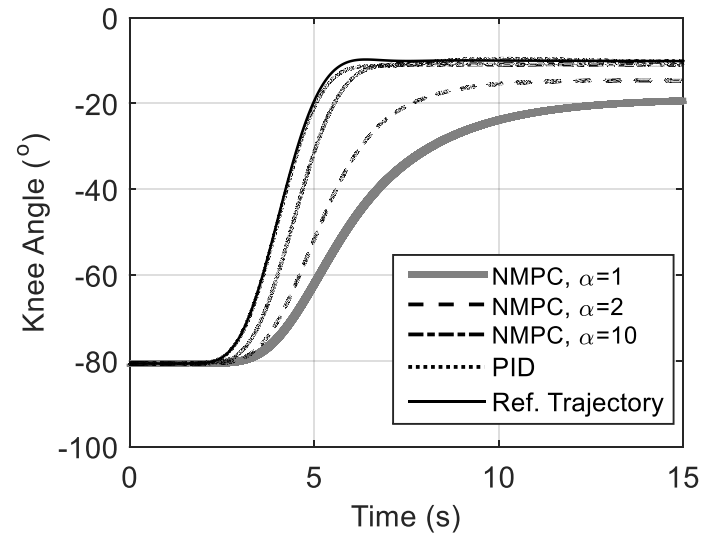

Fig. 3. Comparison of the PID controller with the NMPC with different tracking to effort ratios.

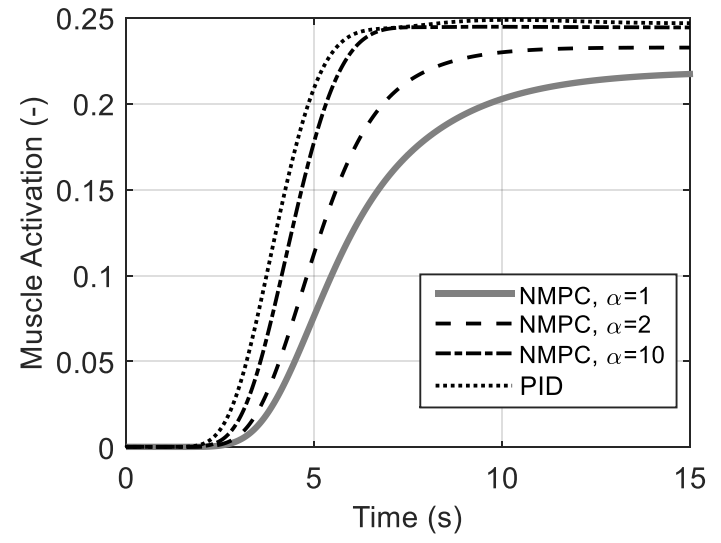

Fig. 4. the required muscle activation corresponding to the NMPC and PID controllers.

Figure 6 shows the effect of prediction horizon length on the tracking performance. As expected, increasing the prediction horizon $(N)$ improves the tracking performance but makes it computationally more expensive. For large systems a trade-off between the trajectory tracking and prediction horizon length is required to ensure the real-time capability of the controller.

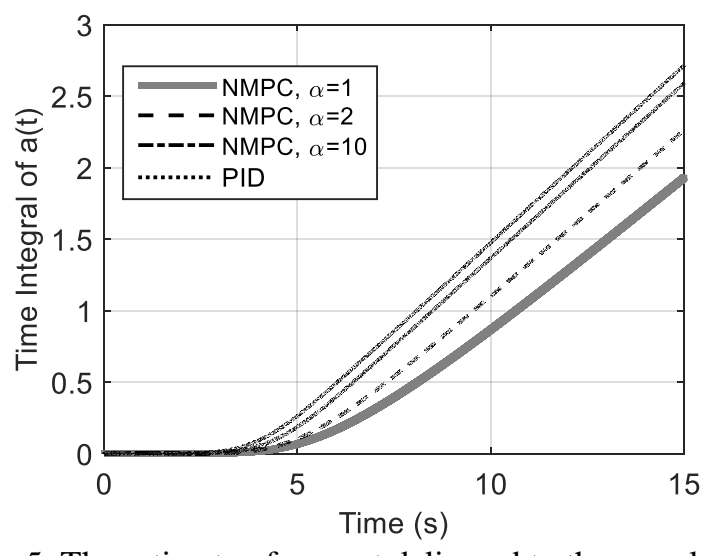

Fig. 5. The estimate of current delivered to the muscle using controllers with different weightings.

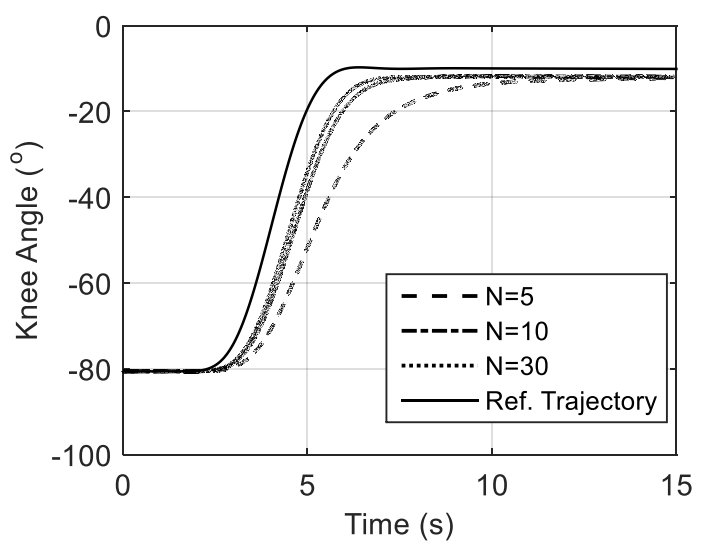

Fig. 6. The effect of prediction horizon length on the trajectory tracking performance.

\section{Conclusion}

The simulation results showed that the Newton/GMRES nonlinear model predictive controller can be successfully used to control the FES system. The proposed FES controller showed satisfactory tracking performance while minimizing the muscle activation. This minimized muscle activation reduces the muscle fatigue, which allows longer rehabilitation sessions. Furthermore, the NMPC controller can be effectively used where the number of stimulators are more than one, allowing optimal recruitments of the muscles. In future work, this controller will be applied to a two degree-of-freedom arm model to simulate the FES therapy for reaching movements.

\section{Acknowledgements}

The authors would like to thank the Natural Sciences and Engineering Research Council of Canada (NSERC) and Canada Research Chairs programs for the financial support of this research.

\section{References}

[1] C. Lynch and M. Popovic, "Functional Electrical Stimulation," in IEEE Control Systems Magazine, vol. 28, no. $2,2008$. 
[2] D. Popović, R. Stein, N. Oğuztöreli, M. Lebiedowska, and S. Jonić, "Optimal control of walking with functional electrical stimulation: a computer simulation study," in IEEE transactions on rehabilitation engineering, vol. 7, no. 1, 1999.

[3] C. Lynch and M. Popovic, "A Comparison of Closed-Loop Control Algorithms for Regulating Electrically Stimulated Knee Movements in Individuals With Spinal Cord Injury," in IEEE Transactions on Neural Systems and Rehabilitation Engineering, vol. 20, no. 4, 2012.

[4] S. Mohammed, P. Poignet, P. Fraisse, and D. Guiraud, "Toward lower limbs movement restoration with input-output feedback linearization and model predictive control through functional electrical stimulation," Control Engineering Practice, vol. 20, no. 2, 2012.

[5] N. Kirsch , N. Alibeji, and N. Sharma , "Nonlinear Model Predictive Control of Functional Electrical Stimulation," in ASME 2015 Dynamic Systems and Control Conference, Columbus, OH, 2015.

[6] M. Ferrarin and A. Pedotti, "The relationship between electrical stimulus and joint torque: a dynamic model," in IEEE Transactions on Rehabilitation Engineering, vol. 8, no. 3, 2000.

[7] T. Ohtsuka, "A continuation/GMRES method for fast computation of nonlinear receding horizon control," Automatica, vol. 40, no. 4, 2004.

[8] C. T. Kelley, Iterative methods for linear and nonlinear equations. SIAM, Philadelphia: North Carolina State University, 1995. 